\title{
On the Use of Minimum and Maximum Attenuation for Retrieving Rainfall Intensity Through Commercial Microwave Links
}

\author{
R. Nebuloni, M. D'Amico, G. Cazzaniga, and C. De Michele
}

\begin{abstract}
Attenuation experienced by commercial microwave links, used for cellular base station backhauling, can be exploited to retrieve rainfall intensity. In this work, we investigate the impact of attenuation data format (minimum and maximum values within a time window of $15 \mathrm{~min}$ ) on the retrieval of rainfall intensity. To this purpose, we exploit the time series of transmitted and received power levels collected by a network of operational microwave links with high temporal resolution (10 s) in Valmalenco (northern Italy).
\end{abstract}

\section{Introduction}

Commercial microwave links (CMLs), widely used to interconnect cellular base stations, have been recently exploited to retrieve the rainfall intensity along the path [1-3]. This technique can be particularly attractive, where rain gauges (RGs) or weather radar or both are not available.

Rainfall rate is estimated from the time series of transmitted and received power levels, which are byproducts of CML networks operation and are used by the network management system to verify link quality. Regrettably, these data sets suffer from two significant limitations, namely, coarse quantization and low temporal resolution: as standards, only the minimum (MIN) and maximum (MAX) values of the transmitted and received power levels, measured during 15 min time slots, are available and stored [4]. The bias introduced by the limitations mentioned previously was investigated in [5] from a purely theoretical point of view.

In this article, we present the results of an experimental investigation aimed at understanding whether the MIN and MAX attenuation values within a time window of $15 \mathrm{~min}$ are sufficient to estimate the mean rainfall intensity over that time window. To this purpose, we exploit data collected by a set of 13 CMLs located in northern Italy. For the duration of the

Manuscript received 31 August 2020.

R. Nebuloni is with Istituto di Elettronica e di Ingegneria dell'Informazione e delle Telecomunicazioni (IEIIT), Consiglio Nazionale delle Ricerche, Piazza Leonardo da Vinci, 32, 20133 Milano, Italy; e-mail: roberto.nebuloni@ieiit.cnr.it.

M. D’Amico is with Dipartimento di Elettronica, Informazione e Bioingegneria (DEIB), Politecnico di Milano, Piazza Leonardo da Vinci, 32, 20133 Milano, Italy; e-mail: michele.damico@polimi.it.

G. Cazzaniga and C. De Michele are with Dipartimento di Ingegneria Civile e Ambientale (DICA), Politecnico di Milano, Piazza Leonardo da Vinci, 32, 20133 Milano, Italy; e-mail: carlo. demichele@polimi.it. experimental campaign, link data storage was modified by the network operator to collect data with high temporal resolution (10 s).

\section{The Experimental Setup}

Measurements presented here were carried out in the Valmalenco valley (a narrow and steep-sided valley of the larger Valtellina valley), an Alpine area in northern Italy where the Mallero River flows. The CML data used in this work are owned by Vodafone Italia and were collected by a network monitoring tool (iVeritas) implemented by SIAE Microelettronica.

Figure 1 shows the location of the links (black solid lines). A total of 13 links are operational in Valmalenco, whose transmission frequencies are between $10.74 \mathrm{GHz}$ and $23.03 \mathrm{GHz}$, while the path lengths are between $2.7 \mathrm{~km}$ and $14.0 \mathrm{~km}$, respectively. Because of the topography, link elevation angles can be as high as $20^{\circ}$. The link terminals are located at heights between $300 \mathrm{~m}$ and more than $2200 \mathrm{~m}$ above sea level. In the study area, there are also 15 RGs owned and operated by the regional environmental protection agency of Lombardy (ARPA Lombardia) and shown in Figure 1 as red triangles. Moreover, two laser disdrometers (DISs) were installed for the duration of the experimental activity (indicated with blue circles and labeled as CAG and PRI). Preliminary results were presented in [6]. In these, the RGs and the DISs were used as the ground truth against which to validate rainfall estimates gathered from CML data.

\section{Data and Methods}

Among the standard data collected by iVeritas, there are the MIN and MAX values of the transmitted and received power levels across each (two-way) link, measured during 15 min time slots; we call this the MIN-MAX format. Specifically, received and transmitted power levels are measured every $200 \mathrm{~ms}$ with a resolution of $0.125 \mathrm{dBm}$, four samples are averaged and quantized with $1 \mathrm{~dB}$ resolution, and only the MIN and MAX values recorded during the $15 \mathrm{~min}$ interval are stored. For this work, iVeritas generated special data, i.e., transmitted and received power levels collected by links 9-13 of Figure 1, once every $10 \mathrm{~s}$.

The procedure for the estimation of the pathaveraged rain rate starting from MIN and MAX measurements consists of five steps:

1. Identifying the outliers; 


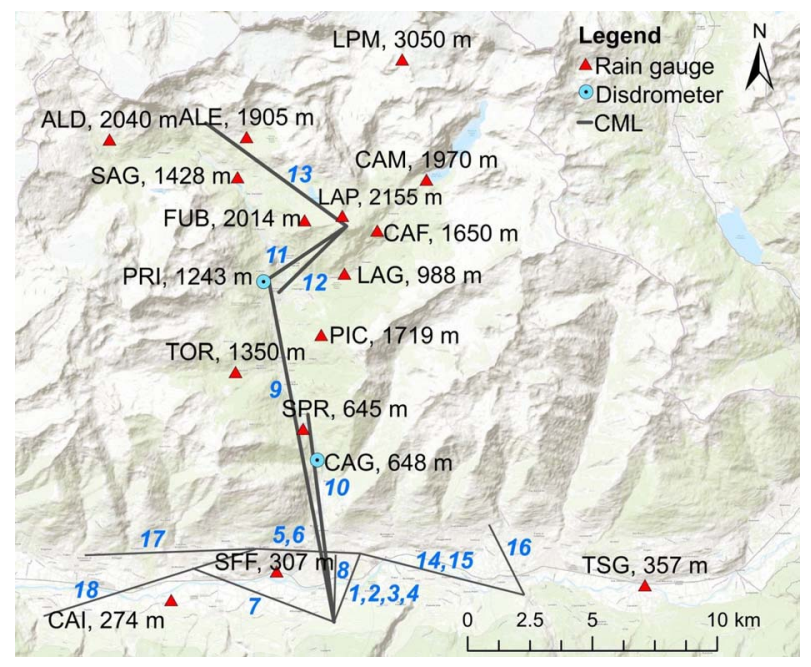

Figure 1. Location of CMLs, RGs and DISs in the Alpine area of Valmalenco and Valtellina (northern Italy). The $10 \mathrm{~s}$ data used in this work are available for links $9,10,11,12$, and 13 .

2. Classifying sampling intervals as wet or dry;

3. Building the baseline;

4. Calculating rain attenuation;

5. Calculating rain intensity.

The procedure is described in details in [6]. Here, we just recall that path-averaged rain rate $R$ is related to rain attenuation $A_{R}$ through the power law relation (also known as the standard International Telecommunication Union Radiocommunication Sector power law formula):

$$
A_{R}=L \kappa R^{\alpha}
$$

where $L$ is the path length, whereas $\kappa$ and $\alpha$ are coefficients dependent on frequency, polarization and link elevation [7]. For links 9-13, the value of the coefficients $\kappa$ and $\alpha$ is shown in Table 1 (for vertical polarization).

Both MIN-MAX and $10 \mathrm{~s}$ data suffer from poor resolution in the signal level, due to the $1 \mathrm{~dB}$ quantization step, whose impact is twofold: it determines the link sensitivity (i.e., its ability to detect light precipitation), and it introduces uncertainties in the measurements. Link sensitivity is the MIN detectable rain rate $R_{\mathrm{MIN}}$, which depends on the error on the measurement of $A_{R}$. For the links in Valmalenco, $R_{\mathrm{MIN}}$ goes from $0.7 \mathrm{~mm} / \mathrm{h}$ to $2.0 \mathrm{~mm} / \mathrm{h}$ (see Table 1 ). Of course, if rain is not uniform along the path, $R_{\text {MIN }}$ increases. The uncertainty due to the quantization step

Table 1. Characteristics of CMLs with $10 \mathrm{~s}$ sampling and values for the parameter in (1)

\begin{tabular}{lccccc}
\hline CML & $\begin{array}{c}\text { Frequency } \\
(\mathrm{GHz})\end{array}$ & $\begin{array}{c}\text { Length } \\
(\mathrm{km})\end{array}$ & $\begin{array}{c}R_{\mathrm{MIN}} \\
(\mathrm{mm} / \mathrm{h})\end{array}$ & $\kappa$ & $\alpha$ \\
\hline 9 & 11.3 & 14.0 & 2.0 & 0.0161 & 1.1702 \\
10 & 18.8 & 8.4 & 0.7 & 0.0842 & 0.9951 \\
11 & 19.2 & 3.9 & 1.4 & 0.0882 & 0.9943 \\
12 & 23.0 & 4.0 & 1.0 & 0.1287 & 0.9663 \\
13 & 18.8 & 7.0 & 1.0 & 0.0842 & 0.9951 \\
\hline
\end{tabular}
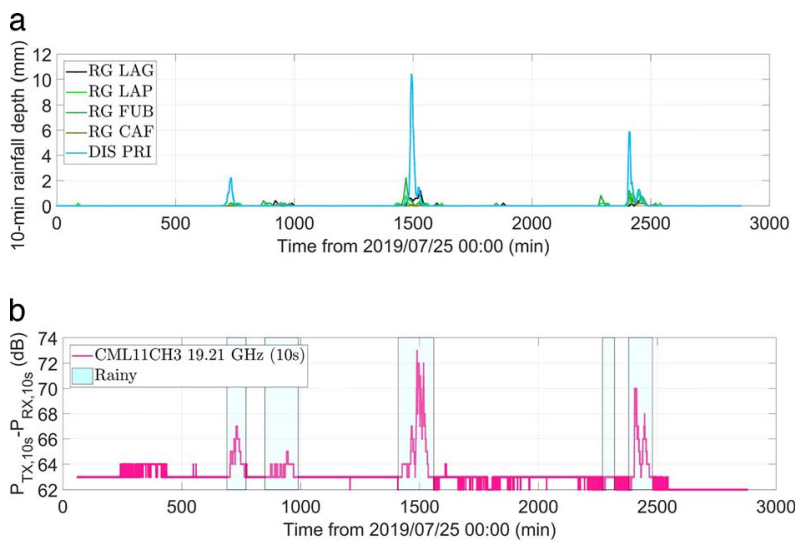

Figure 2. (a) Rainfall depth from precipitation sensors in the proximity of CML 11 and (b) corresponding difference between transmitted and received power by CML 11 during event 2. The shaded areas in (b) are the precipitation episodes, as identified and described in Section 3.

is larger for low attenuation values, i.e., low rain intensities, short links, and low frequencies.

An important issue is the correct classification of each $15 \mathrm{~min}$ time interval as wet (or dry), i.e., characterized by the presence (or absence) of rainfall [8]. In this work, dry or wet identification is based on nearby precipitation sensors (i.e., within $3 \mathrm{~km}$ from the closest CML point). For instance, CML 11 in Figure 1 is associated with four RGs, namely, FUB (at a distance of $1.3 \mathrm{~km}$, calculated as the average of the distances to the terminals and the midway point), LAG $(1.8 \mathrm{~km})$, LAP $(2.1 \mathrm{~km})$, PRI $(2.1 \mathrm{~km})$, and the DIS CAF (2.9 km). RGs are tipping buckets with a $0.2 \mathrm{~mm}$ resolution and returning time series of rainfall depth over $10 \mathrm{~min}$ intervals, whereas the two DISs have a 1 min sampling time. The rationale for rain event identification consists of first thresholding the RG and DIS time series of rainfall depth and grouping positives into a single wet segment if there are periods of zero rainfall not longer than $10 \mathrm{~min}$ in between. An event shall last $30 \mathrm{~min}$ at least, and the MAX rain intensity shall be $2 \mathrm{~mm} / \mathrm{h}$ at least. However, a CML is not a point sensor. Instead, it is a distributed sensor that captures rainfall falling along its path. Therefore, an "event," as detected by a CML is identified by a slightly more complex algorithm. To this aim, we built the sample-by-sample MAX rainfall depth among the ones of the associated RG and DIS and thresholded this new time series. Figure 2 shows an example of this procedure. The peaks in Figure $2 b$, which correspond to the rainy periods detected by the CML, are well identified. The quantity displayed is the bare difference between transmitted and received power (both in decibels with reference to $1 \mathrm{~mW}$ ), which undergoes the procedure described previously to extract the time series of rain attenuation and, finally, rainfall intensity.

Through a simple manipulation of $10 \mathrm{~s}$ data, we can reproduce some common formats for available CML data, such as 15 min MIN-MAX, which is used by a number of investigators $[4,5]$. The time axis of 10 


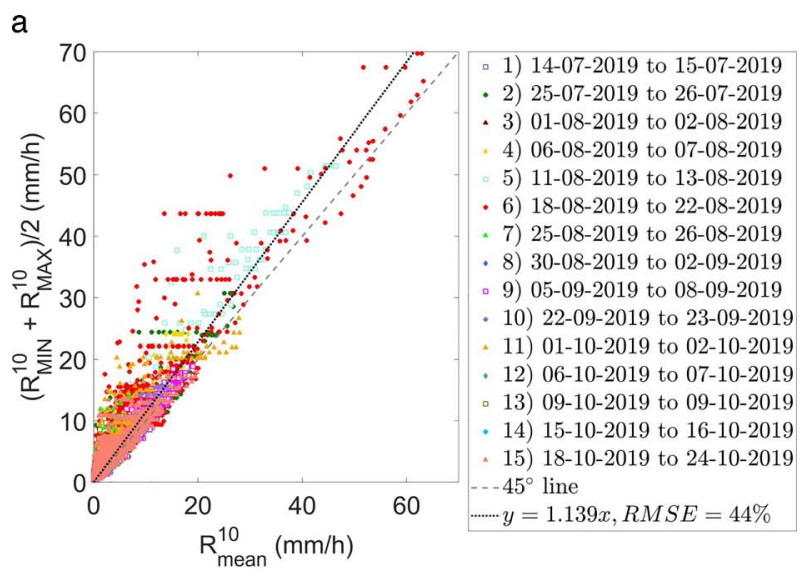

b

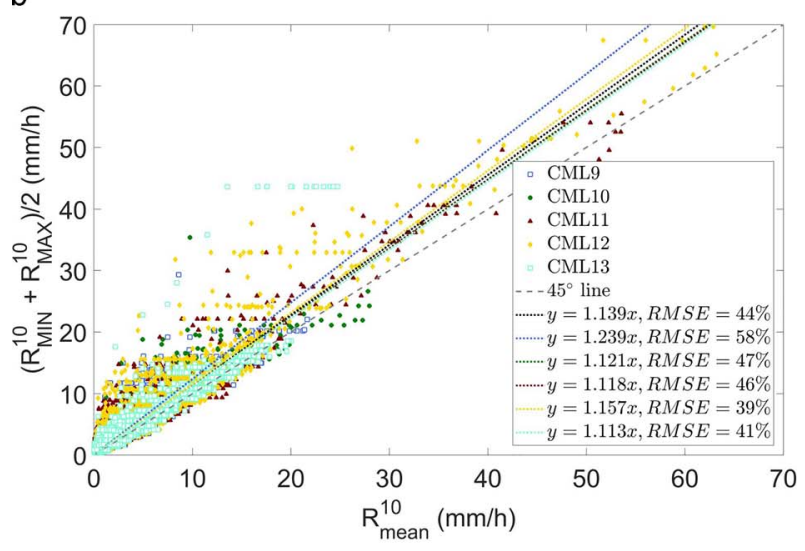

C

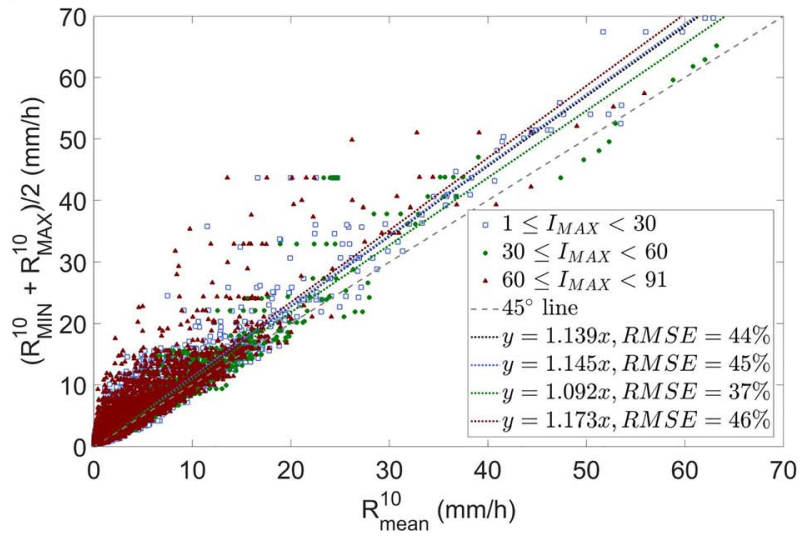

Figure 3. Mean rainfall intensity against estimate of the mean value on the basis of the MIN and MAX values in 15 min windows, as gathered from $10 \mathrm{~s} \mathrm{CML} \mathrm{data.} \mathrm{Measurements} \mathrm{points} \mathrm{have} \mathrm{been} \mathrm{grouped}$ according to (a) event, (b) CML, and (c) position of the rainfall peak within every window. Linear best fit curves are shown, as well for the entire population and the different sets of points, for (b) and (c) only.

s data has been divided into partially overlapping windows of duration $\Delta T=15 \mathrm{~min}$ each. Only windows fully included in the previously identified events are considered. The 15 min mean of the quantity of interests (e.g., rainfall intensity or rain attenuation) can be extracted and subsequently compared with its MIN, MAX, or a suitable combination of them to assess whether one can obtain a proper estimate of the mean by the extremes only.

It is expected that the characteristics of the histograms drawn from the 90 samples within each window will be dependent on two factors (among others): rainfall peak and peak position within the window. The dependence of results on peak position can be investigated by using overlapping windows (in this work, consecutive $15 \mathrm{~min}$ windows are shifted by 1 min). The analysis has been carried over a set of 15 rainfall events, collected from July 2019 to October 2019 . On the average, approximatively $250 \mathrm{~h}$ of rainfall were processed.

\section{Results}

Let us consider estimates of the mean $15 \mathrm{~min}$ rainfall intensity obtained from MIN and MAX values of attenuation. We can either calculate the average value of rain attenuation from MIN and MAX values and then convert it into rainfall rate according to (1) or calculate MIN and MAX values of rainfall rate from the corresponding attenuation values and then make the arithmetic mean of them. The former procedure, in principle, introduces a bias, due to the nonlinearity of (1), which depends on the link frequency. For this reason, in the following, we consider the latter procedure and denominate it as MIN-MAX millimeter per hour estimator of mean rainfall. Figure 3 shows the scatterplot of the MIN-MAX millimeter per hour estimator against the $15 \mathrm{~min}$ mean rainfall rate for all events, calculated by first converting the $10 \mathrm{~s}$ attenuation in rainfall rate and then making the average of these. The measurement points are grouped into subsets, according to three different features: events in Figure 3a, CMLs in Figure $3 \mathrm{~b}$, and position ( $I_{\text {MAX }}$, multiple of $10 \mathrm{~s}, 1 \leq I_{\mathrm{MAX}} \leq 90$ ) of the rainfall peak within every (15 min) sampling interval in Figure 3c, respectively.

The best fit straight line $y=m x$ is plotted for the entire data set and for Figures $3 b$ and $3 c$, for each subset, as well as the 1:1 straight line. Figure $3 a$ highlights the 15 events with different colors. As somewhat expected, the MIN-MAX millimeter per hour estimator produces a slight overestimate of the actual mean rainfall intensity, which is around $14 \%$, considering the entire population. However, the root mean square error (RMSE) is significant (in excess of $40 \%$ ). The best fit slope parameter is event dependent.

In Figure 3b, the colors identify the five CMLs used in this work. The best fit slope parameter is similar for CMLs 10-13, whereas it is significantly different for CML 9, operating at a lower frequency and being much longer than the others. We verified that using an estimator on the basis of attenuation, the slope would be 1.34 instead of the actual 1.24, which can be ascribed to the value of the coefficient $\alpha$ at $11.3 \mathrm{GHz}$ (see Table 1). The impact of data collected by CML 9 on the calculation of the slope and of the RMSE is negligible, due to the large number of measurements falling below 


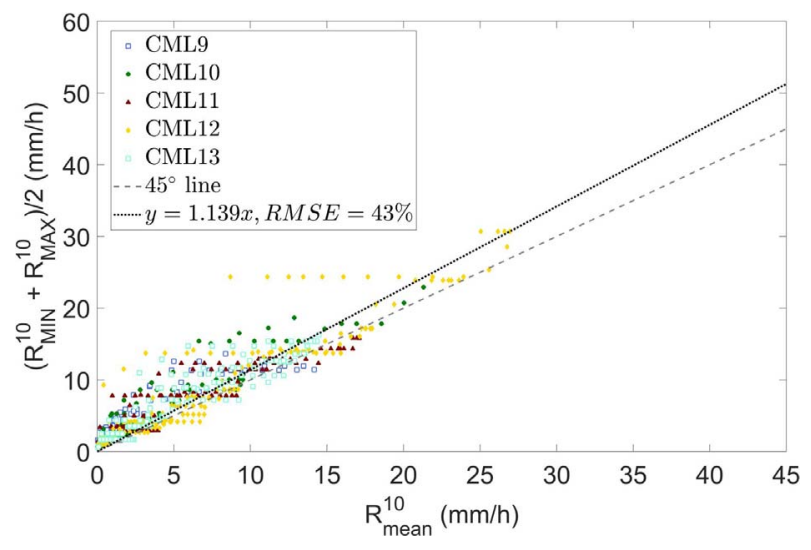

Figure 4. As in Figure 3b, this time is for the event of July 25-26, 2019.

link sensitivity; hence, even discarding CML 9 data, the previously mentioned numbers do not change.

In Figure 3c, colors highlight the position of the rainfall intensity peak within every $15 \mathrm{~min}$ window (i.e., 90 samples). There is not a clear dependence of the relationship between mean and MIN-MAX millimeter per hour estimate on the position of the rainfall peak.

The previously mentioned scatterplots highlight a few interesting features: there are aligned points along the horizontal direction, e.g., events 2,4 , and 6 , with $y$ values of about $22 \mathrm{~mm} / \mathrm{h}, 24 \mathrm{~mm} / \mathrm{h}, 32 \mathrm{~mm} / \mathrm{h}$, and 44 $\mathrm{mm} / \mathrm{h}$, respectively. For sake of clarity, Figure 4 shows the scatterplot relative to event 2 only; the anomalous pattern corresponds to the measurement points of a single link, CML 12.

Figure 5 shows a particular of the time series of rainfall intensity for CML 12 during event 2, specifically, at the occurrence of a convective burst up to 50 $\mathrm{mm} / \mathrm{h}$, which explains the reason of this behavior. As far as the peak is within the 15 min window, the MINMAX decibel estimate is basically unchanged, whereas the mean value fluctuates due to random variations of the rainfall process in the period. Part of the scatter of the MIN-MAX decibel estimate, especially at large values of MAX rainfall intensity, is explained by signal undersampling due to MIN-MAX logging. A possible improvement of the estimator is to make the MINMAX average dependent on the actual value of MAX intensity in the window.

\section{Conclusions}

Rainfall retrievals obtained from the opportunistic usage of data available from CML networks may contribute to the two-dimensional rainfall field reconstruction at ground level. As CML data often undergo 15 min MIN-MAX logging, in this article, we answered the question whether the MIN and MAX values of attenuation are sufficient to provide an estimate of the mean rainfall intensity. To this aim, we used CML data with high temporal resolution (10 s) and simulated the 15 min MIN-MAX logging process. Finally, we calculated

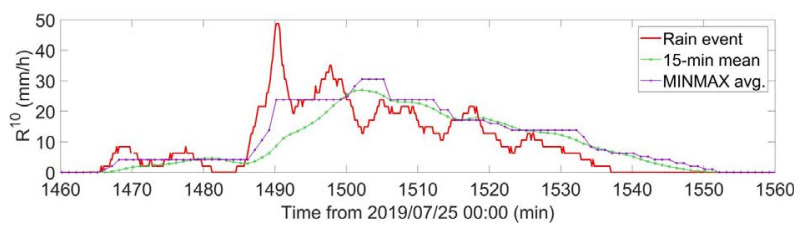

Figure 5. Time series of rainfall intensity for CML 12 of Figure 4. In red is the rainfall estimate from $10 \mathrm{~s}$ data, in green, the $15 \mathrm{~min}$ rainfall average (the value at time $t$ is obtained by averaging raw data from the previous $15 \mathrm{~min}$ ), and, finally, in violet, the MIN-MAX decibel average.

the mean by a simple two-step rule (the MIN-MAX millimeter per hour estimator), which is based on averaging MIN and MAX rainfall rate and dividing by 1.14. This estimator provides an unbiased estimate of the mean over the considered data set. The percentage of the RMSE is rather large, however, due to the random nature of the rainfall process. A better estimator should rely on a model of the probability distribution function of rainfall in the observation window.

\section{Acknowledgments}

This work has been carried out under the MOPRAM project. The authors are grateful to Fondazione Cariplo for funding the project, SIAE Microelettronica for the technical support, and Vodafone Italia for providing CML data.

\section{References}

1. A. R. Rahimi, A. R. Holt, G. J. G. Upton, and R. J. Cummings, "Use of Dual-Frequency Microwave Links for Measuring Path-Averaged Rainfall", Journal of Geophysical Research - Atmospheres, 108, 2003.

2. H. Messer, A. Zinevich, and P. Alpert, "Environmental Monitoring by Wireless Communication Networks," Science, 312, 5774, May 2006, p. 713.

3. H. Messer, "Rainfall Monitoring Using Cellular Networks," IEEE Signal Processing Magazine, 24, 3, May 2007, pp. 144-142.

4. A. Overeem, H. Leijnse, and R. Uijlenhoet, "Measuring Urban Rainfall Using Microwave Links From Commercial Cellular Communication Networks," Water Resources Research, 47, 12, December 2011. doi:10.1029/ 2010WR010350, pp. 1-16.

5. J. Ostrometzky, A. Eshely, P. Alperty, and H. Messer, "Induced Bias in Attenuation Measurements Taken From Commercial Microwave Links," 2017 IEEE International Conference on Acoustics, Speech, and Signal Processing, New Orleans, LA, USA, March 5-9, 2017, pp. 3744-3748.

6. R. Nebuloni, D. Tagliaferri, M. D’Amico, A. Sileo, G. Cazzaniga, et al., "Rainfall Retrieval Through Commercial Microwave Links in Valmalenco (North Italy)," ThirtyThird General Assembly and Scientific Symposium of the International Union of Radio Science, Rome, Italy, August 29-September 5, 2020, pp. 1-4.

7. International Telecommunication Union, Specific Attenuation Model for Rain for Use in Prediction Methods, Geneva, Switzerland, 2005.

8. M. Schleiss and A. Berne, "Identification of Dry and Rainy Periods Using Telecommunication Microwave Links," IEEE Geoscience and Remote Sensing Letters, 7, 3, March 2010, pp. 611-615. 